\title{
On the planar Schrödinger-Poisson system with zero mass potential
}

Fangfang Liao ${ }^{1}$ and Xiaoping Wang ${ }^{1}$

${ }^{1}$ XiangNan University

July 18, 2021

\begin{abstract}
In this paper, we prove that the following planar Schrödinger-Poisson system with zero mass $-\Delta \mathrm{u}+\varphi \mathrm{u}=\mathrm{f}(\mathrm{u}), \mathrm{x}[?] \mathrm{R}^{\wedge} 2, \Delta \varphi=$ $2 \pi \mathrm{u}^{\wedge} 2, \mathrm{x}[?] \mathrm{R}^{\wedge} 2$, admits a nontrivial radially symmetric solution under weaker assumptions on $\mathrm{f}$ by using some new analytical approaches.
\end{abstract}

\section{Hosted file}

Liao-Wang-2103.pdf available at https://authorea.com/users/426247/articles/530805-on-theplanar-schr\%C3\%B6dinger-poisson-system-with-zero-mass-potential

\section{Hosted file}

Liao-Wang-2103.tex available at https://authorea.com/users/426247/articles/530805-on-theplanar-schr\%C3\%B6dinger-poisson-system-with-zero-mass-potential 